\title{
Transition from decelerated to accelerated cosmic expansion in braneworld universes
}

\author{
J. Ponce de Leon* \\ Laboratory of Theoretical Physics, Department of Physics \\ University of Puerto Rico, P.O. Box 23343, Rio Piedras, \\ PR 00931, USA
}

April 1, 2005

\begin{abstract}
Braneworld theory provides a natural setting to treat, at a classical level, the cosmological effects of vacuum energy. Non-static extra dimensions can generally lead to a variable vacuum energy, which in turn may explain the present accelerated cosmic expansion. We concentrate our attention in models where the vacuum energy decreases as an inverse power law of the scale factor. These models agree with the observed accelerating universe, while fitting simultaneously the observational data for the density and deceleration parameter. The redshift at which the vacuum energy can start to dominate depends on the mass density of ordinary matter. For $\bar{\Omega}_{m}=0.3$, the transition from decelerated to accelerated cosmic expansion occurs at $z_{T} \approx 0.48 \pm 0.20$, which is compatible with SNe data. We set a lower bound on the deceleration parameter today, namely $\bar{q}>-1+3 \bar{\Omega}_{m} / 2$, i.e., $\bar{q}>-0.55$ for $\bar{\Omega}_{m}=0.3$. The future evolution of the universe crucially depends on the time when vacuum starts to dominate over ordinary matter. If it dominates only recently, at an epoch $z<0.64$, then the universe is accelerating today and will continue that way forever. If vacuum dominates earlier, at $z>0.64$, then the deceleration comes back and the universe recollapses at some point in the distant future. In the first case, quintessence and Cardassian expansion can be formally interpreted as the low energy limit of our model, although they are entirely different in philosophy. In the second case there is no correspondence between these models and ours.
\end{abstract}

PACS: 98.80.-k, 98.80.Es, 98.80.Jk, 04.20.-q, 04.50.+h, 04.20.Cv

Keywords: cosmic accelerated expansion - brane theory - cosmology: theory - Variable fundamental constants - Cardassian expansion - dark matter

*E-Mail: jponce@upracd.upr.clu.edu,jpdel@astro.uwaterloo.ca 


\section{Introduction}

It is now widely accepted that the present universe is accelerating and spatially flat. Evidence in favor of this is provided by observations of high-redshift supernovae Ia [1]-[] , as well as other observations, including the cosmic microwave background and galaxy power spectra $7-13$.

Since ordinary matter is gravitationally attractive, one can conclude that the source of cosmic acceleration is some kind of unknown matter, which violates the strong energy condition. According to dynamical mass measurements, the total amount of ordinary matter in the universe, including dark matter, can only account for $30 \%$ of the critical density. Thus, the other $70 \%$ corresponds to a mysterious form of matter, usually called dark energy, which remains unclustered on all scales where gravitational clustering of ordinary matter is seen.

Therefore, in the past few years an active field of research has been the discovery of models of the universe in which the expansion is accelerating. The simplest candidate for dark energy is the cosmological constant $\Lambda_{(4)}$ 14-15. In this approach, $\Lambda_{(4)}$ is introduced by "hand" as a parameter in Einstein's theory of gravity. However, if $\Lambda_{(4)}$ remains constant one faces the problem of fine-tuning or "cosmic coincidence problem" [16], which refers to the coincidence that $\rho_{v a c}$ and $\rho_{m}$ are of the same order of magnitude today.

A phenomenological solution to this problem is to consider a time dependent cosmological term, or an evolving scalar field known as quintessence [16-[19]. A variable $\Lambda_{(4)}$, as well as quintessence, can be modeled as the energy of a slowly evolving cosmic scalar field $\phi$ with an appropriate self-interaction potential $V(\phi)$ to account for the evolution of the universe. The abundance of quintessence models is due to the fact that for any given scale factor $a(t)$ and some known forms of energy density $\rho_{\text {known }}(t)$ (made of radiation, matter, etc), it is always possible to find a $V(\phi)$ that explains the observations [20].

Alternative explanations for the acceleration of the universe, beyond dark energy, include phantom energy [21][24, certain modifications of general relativity [25]-33], the gravitational leakage into extra dimensions [34- 36], Chaplygin gas 37-39] as well as Cardassian models [40- 41]. More recently, in a series of papers Vishwakarma [42], 43 and Vishwakarma and Singh [44] argue that present observations can successfully be explained by a decelerating model of the universe in the mainstream cosmology, without invoking dark matter or dark energy.

In the present work, we study the accelerated cosmic expansion as a consequence of embedding our observable universe as a brane in extra dimensions. Our motivation is that braneworld theory provides a natural setting to treat, at a classical level, the cosmological effects of vacuum energy. Firstly, the theory links the vacuum energy to the fundamental quantities $\Lambda_{(4)}$ and $G$. Secondly, in this theory the vacuum energy is completely determined by the geometry in $5 D$ through Israel's boundary conditions [45-47]. Said another way, in brane theory the cosmological term is determined by the bulk geometry and cannot be put by hand as in Einstein's theory. Thirdly, non-static extra dimensions can generally lead to a variable vacuum energy [48, and consequently variable $\Lambda_{(4)}$ and $G$, which in turn may explain the present accelerated cosmic expansion.

In fact, in recent papers [49]-[50] we showed that braneworld models with variable vacuum energy (and zero or negative bulk cosmological constant) agree with the observed accelerating universe, while fitting simultaneously the observational data for the density and deceleration parameter. In those papers we were mostly interested in the general behavior of the models. We obtained precise constrains on the cosmological parameters as well as demonstrated that the "effective" equation of state of the universe can, in principle, be determined by measurements of the deceleration parameter alone.

In this paper we are interested in more subtle details. For example, we would like to know the redshift of transition from deceleration to acceleration. Namely, based on our model, can we predict, or at least narrow down, the redshift interval for the transition? What is the time at which the vacuum energy should start to dominate in order to explain the observed cosmic acceleration? How is it related to the current mass density of the universe? Does the present deceleration parameter depend on it? What is the future evolution of the universe? Will it continue to accelerate forever?

In the scenario discussed here, the acceleration of the universe is related not only to the variation of vacuum energy and cosmological term, but also to the time evolution of $G$ and, possibly, to the variation of other fundamental "constants" as well.

The paper is organized as follows. In section 2 we give a brief summary of the equations for homogeneous cosmologies in $5 D$ based on braneworld theory. In section 3 we show how to incorporate a varying vacuum energy 
into the scheme. Observational constraints on the model are discussed in section 4 . In section 5 we discuss the transition from deceleration to acceleration.

We show that the crossover point strongly depends on the energy density of ordinary matter. The possible values of the redshift of transition are spread over a finite interval, regardless of the moment in time when vacuum starts to dominate over ordinary matter. This is a clear indication that the effects of vacuum rapidly decrease with the increase of $z$. We also show that the value of the deceleration parameter today is bounded bellow.

We discuss the cosmological parameters for the case where the matter density today is one-third of the critical density. We find that the future evolution of the universe crucially depends on the time when vacuum starts to dominate over ordinary matter. Namely, if the vacuum contribution starts to dominate only recently, at an epoch $z<0.64$, then the universe is accelerating today and will continue that way forever. But, if the vacuum dominates earlier, at $z>0.64$, then the deceleration comes back and the universe recollapses at some point in the distant future.

Finally, in section 6 we give a summary and discussion. We show that the original Cardassian expansion, proposed by Freese and Lewis [40, as well as dark energy models (with constant $w_{X}$ ) can be interpreted as the low energy limit of our ever-expanding models.

\section{Homogeneous cosmology in $5 D$}

In order to facilitate the discussion, and set the notation, we start with a brief summary of the pertinent ideas and equations in the braneworld scenario. In this scenario our homogeneous and isotropic universe is envisioned as a singular hypersurface embedded in a five-dimensional manifold with metric

$$
d \mathcal{S}^{2}=n^{2}(t, y) d t^{2}-a^{2}(t, y)\left[\frac{d r^{2}}{\left(1-k r^{2}\right)}+r^{2}\left(d \theta^{2}+\sin ^{2} \theta d \phi^{2}\right)\right]-\Phi^{2}(t, y) d y^{2},
$$

where $t, r, \theta$ and $\phi$ are the usual coordinates for a spacetime with spherically symmetric spatial sections and $k=$ $0,+1,-1$. The metric is a solution of the five-dimensional Einstein equations

$$
{ }^{(5)} G_{A B}={ }^{(5)} R_{A B}-\frac{1}{2} g_{A B}{ }^{(5)} R=k_{(5)}^{2}{ }^{(5)} T_{A B},
$$

where ${ }^{(5)} T_{A B}$ is the five-dimensional energy-momentum tensor and $k_{(5)}$ is a constant introduced for dimensional considerations.

The energy-momentum tensor on the brane $\tau_{\mu \nu}$ is separated in two parts,

$$
\tau_{\mu \nu}=\sigma g_{\mu \nu}+T_{\mu \nu}
$$

where $\sigma$ is the tension of the brane in $5 D$, which is interpreted as the vacuum energy of the braneworld, and $T_{\mu \nu}$ represents the energy-momentum tensor of ordinary matter in $4 D$.

There are two assumptions relating the physics in $4 D$ to the geometry of the bulk. They are: (i) the bulk spacetime possesses $\mathbf{Z}_{\mathbf{2}}$ symmetry about the brane, and (ii) the brane is embedded in an Anti-de Sitter bulk, i.e., ${ }^{(5)} T_{A B}$ is taken as

where $\Lambda_{(5)}<0$.

$$
{ }^{(5)} T_{A B}=\Lambda_{(5)} g_{A B},
$$

As a consequence of the first assumption, the matter and vacuum energy density in $4 D$ become completely determined by the geometry in $5 D$ through Israel's boundary conditions. Namely, for a perfect fluid

$$
T_{\mu \nu}=(\rho+p) u_{\mu} u_{\nu}-p g_{\mu \nu},
$$

with

$$
p=\gamma \rho,
$$

the matter density is given by 48

$$
\rho=\frac{(-2)}{\left.k_{(5)}^{2}(\gamma+1) \Phi\right|_{\text {brane }}}\left[\frac{a^{\prime}}{a}-\frac{n^{\prime}}{n}\right]_{\text {brane }} .
$$


and the vacuum density is

$$
\sigma=\frac{(-2)}{\left.k_{(5)}^{2}(\gamma+1) \Phi\right|_{\text {brane }}}\left[(3 \gamma+2) \frac{a^{\prime}}{a}+\frac{n^{\prime}}{n}\right]_{\text {brane }},
$$

where a prime denotes derivative with respect to the extra variable $y$.

The second assumption has two important consequences. Firstly, since ${ }^{(5)} T_{\mu 4}=0$, it follows that the energymomentum tensor on the brane $\tau_{\mu \nu}$ is a conserved quantity, viz.,

$$
\tau_{\mu ; \nu}^{\nu}=0 .
$$

Secondly, the field equations (2) admit a first integral, namely,

$$
\left(\frac{\dot{a}}{a n}\right)^{2}=\frac{k_{(5)}^{2} \Lambda_{(5)}}{6}+\left(\frac{a^{\prime}}{a \Phi}\right)^{2}-\frac{k}{a^{2}}+\frac{\mathcal{C}}{a^{4}}
$$

where $\mathcal{C}$ is a constant of integration which arises from the projection of the Weyl curvature tensor of the bulk on the brane. Evaluating (10) at the brane, which is fixed at some $y=y_{\text {brane }}=$ const, as well as using (7) and (8), we obtain the generalized Friedmann equation, viz.,

$$
3\left(\frac{\dot{a}_{0}}{a_{0}}\right)^{2}=\Lambda_{(4)}+8 \pi G \rho+\frac{k_{(5)}^{4}}{12} \rho^{2}-\frac{3 k}{a_{0}^{2}}+\frac{3 \mathcal{C}}{a_{0}^{4}},
$$

where $a_{0}(t)=a\left(t, y_{\text {brane }}\right)$, and

$$
\begin{gathered}
\Lambda_{(4)}=\frac{1}{2} k_{(5)}^{2}\left(\Lambda_{(5)}+\frac{k_{(5)}^{2} \sigma^{2}}{6}\right), \\
8 \pi G=\frac{k_{(5)}^{4} \sigma}{6} .
\end{gathered}
$$

These quantities are interpreted as the net cosmological term and gravitational coupling in 4 dimensions, respectively.

Equation (11) contains two novel features; it relates the fundamental quantities $\Lambda_{(4)}$ and $G$ to the vacuum energy, and carries higher-dimensional modifications to the Friedmann-Robertson-Walker (FRW) cosmological models of general relativity. Namely, local quadratic corrections via $\rho^{2}$, and the nonlocal corrections from the free gravitational field in the bulk, transmitted by the dark radiation term $\mathcal{C} / a^{4}$.

Except for the condition that $n=1$ at the brane, the generalized Friedmann equation (11) is valid for arbitrary $\Phi(t, y)$ and $n(t, y)$ in the bulk [47. This equation allows us to examine the evolution of the brane without using any particular solution of the five-dimensional field equations. In what follows we will omit the subscript 0 .

\section{$3 \quad$ Variable vacuum energy}

In equation (11), $G$ and $\Lambda_{(4)}$ are usually assumed to be "truly" constants. However, the vacuum energy density $\sigma$ does not have to be a constant. From (8) it follows that $\sigma$ depends on the details of the model. Indeed, we have recently shown 48 that there are several models, with reasonable physical properties, for which a variable $\Phi$ induces a variation in the vacuum energy $\sigma$.

\subsection{Variable vacuum: an example}

As an illustration, let us consider the class of solutions to the field equations (2) for which the metric coefficients in (11) are separable functions of their arguments. For this class of solutions, without loss of generality we can set

$$
n=n(y), \quad a(t, y)=\tilde{a}(t) Y(y), \quad \Phi=\Phi(t) .
$$


From $G_{04}=0$ it follows that

$$
\left(\frac{n^{\prime}}{n}\right)=\zeta\left(\frac{Y^{\prime}}{Y}\right), \quad \frac{\dot{\Phi}}{\Phi}=(1-\zeta) \frac{\dot{\tilde{a}}}{\tilde{a}}
$$

where $\zeta$ is a separation constant. Consequently,

$$
\Phi(t)=A \tilde{a}^{(1-\zeta)},
$$

where $A$ is a constant of integration. Thus, for any $\zeta \neq 1, \Phi$ is a variable function of $t$. In what follows, for simplicity of the notation, instead of $\zeta$ we will use $\beta=-(\zeta+2) / 3$ (or $\zeta=-3 \beta-2)$. With this notation we have $\Phi(t)=A \tilde{a}^{3(\beta+1)}$. Substituting this expression into [8] we find

$$
\sigma=\frac{D(\gamma-\beta)}{(\beta+1) a^{3(\beta+1)}}
$$

where we have introduced the constant $D \equiv-6(\beta+1) Y_{\text {brane }}^{\prime} Y_{\text {brane }}^{3 \beta+2} / A(\gamma+1) k_{(5)}^{2}$. This epitomizes the general situation where $\sigma$ is a function of time. It is worth noting that only the assumption of separability of the bulk metric (1) underlies equation (17), i.e., we do not need to know the details of the solution in the five-dimensional bulk.

From a physical point of view, the vacuum energy (17) implies $(\dot{G} / G)=-3(\beta+1) H$. Conversely, if we extrapolate the present limit $|\dot{G} / G|=|g| H$, and assume that $g$ is a constant $^{1}$, then we obtain a cosmological model where the vacuum energy is given exactly by (17), with $g=-3(\beta+1)$. This model is consistent with the observed acceleration and flatness of our universe 49 .

\subsection{Our model}

In general, for variable vacuum energy, the conservation equations (9) for a perfect fluid which satisfies (6), yield

$$
\dot{\rho}+3 \rho(\gamma+1) \frac{\dot{a}}{a}=-\dot{\sigma}
$$

From which it follows that

$$
\rho=\frac{C}{a^{3(\gamma+1)}}-\frac{1}{a^{3(\gamma+1)}} \int a^{3(\gamma+1)} d \sigma
$$

where $C$ is a constant of integration. For the case of constant $\sigma$, we recover the familiar relationship between the matter energy density and the expansion factor $a$, viz.,

$$
\rho=\frac{C}{a^{3(\gamma+1)}}
$$

The second term in (19) is the contribution associated with the variation of vacuum. The variation of the vacuum energy is deeply rooted in fundamental physics. The simplest microphysical model for a variable $\sigma$, as well as for $\Lambda_{(4)}$ and quintessence, is the energy associated with a slowly evolving cosmic scalar field $\phi$ with some self-interaction potential $V(\phi)$ minimally coupled to gravity. The potentials are suggested by particle physics, but in principle $V(\phi)$ can be determined from supernova observations [51]-[53].

In this paper, instead of constructing a field theory for the time evolution of the vacuum energy ${ }^{2}$, we employ our previous example as a guide. Namely, if during the expansion of the universe $\sigma$ decays as in (17), then from (19) it follows that

$$
\rho=\frac{C}{a^{3(\gamma+1)}}+\frac{D}{a^{3(\beta+1)}}
$$

\footnotetext{
${ }^{1}$ The physical meaning of this assumption is that the variation of $g$ is much "slower" than that of $H$ and $G$. Namely, $|\dot{g} / g|<<|\dot{H} / H|$, $|\dot{g} / g|<<|\dot{G} / G|$

${ }^{2}$ According to Padmanabhan $[20$ it is trivial to choose the "appropriate" potential $V(\phi)$ such that we can explain the observations, for any given pair $a(t)$ and $\rho(t)$
} 
Now, from the conservation equation (18) we get

$$
\sigma=\sigma_{0}+\frac{D(\gamma-\beta)}{(\beta+1) a^{3(\beta+1)}}
$$

where $\sigma_{0}$ is a constant of integration. We will assume this form, with $\sigma_{0} \neq 0$, for the vacuum energy ${ }^{3}$. It reduces to the case of constant vacuum energy $\sigma=\sigma_{0}$, for $\beta=\gamma$. However, for $\beta \neq \gamma$ it generates models with variable $\sigma, G$ and $\Lambda_{(4)}$. We immediately notice that the positivity of $G=k_{(5)}^{4} \sigma / 6$ requires $\beta<\gamma$.

\subsubsection{The effective density}

The total energy density of the universe, $\rho_{\text {total }}=\sigma+\rho$, can be written as

$$
\rho_{\text {total }}=\sigma_{0}+\rho_{\text {eff }},
$$

where we have introduced the "effective" density $\rho_{\text {eff }}$ as

$$
\rho_{e f f}=\frac{C}{a^{3(\gamma+1)}}+\left(\frac{\gamma+1}{\beta+1}\right) \frac{D}{a^{3(\beta+1)}} .
$$

This effective density is the one that drives the evolution of the universe. Indeed, in the present model the generalized Friedmann equation becomes

$$
3\left(\frac{\dot{a}}{a}\right)^{2}=\frac{1}{2} k_{(5)}^{2}\left(\Lambda_{(5)}+\frac{k_{(5)}^{2}}{6} \sigma_{0}^{2}\right)+\frac{k_{(5)}^{4} \sigma_{0}}{6} \rho_{\text {eff }}+\frac{k_{(5)}^{4}}{12} \rho_{\text {eff }}^{2}-\frac{3 k}{a^{2}}+\frac{3 \mathcal{C}}{a^{4}} .
$$

We note that, distinct from (21), the vacuum contribution in the effective density (24) is multiplied by the factor $(\gamma+1) /(\beta+1)$, which is larger than 1 , because $\beta<\gamma$.

In what follows we will set $\gamma=0$, in view of the fact that our universe is matter-dominated $(p=0)$. In addition, we can set $k=0$, because astrophysical data from BOOMERANG 54 and WMAP [12 indicate that our universe is flat. Also we can consider $\mathcal{C}=0$, since the constant $\mathcal{C}$, which is an effective radiation term related to the bulk Weyl tensor, is constrained to be small enough at the time of nucleosynthesis and it should be negligible today. Also, in order to avoid an exponential expansion of the universe in its asymptotic limit, we assume

$$
\Lambda_{(5)}+\frac{k_{(5)}^{2}}{6} \sigma_{0}^{2}=0
$$

Thus, in the case under consideration the evolution of the universe will be governed by

$$
3\left(\frac{\dot{a}}{a}\right)^{2}=\frac{k_{(5)}^{4} \sigma_{0}}{6} \rho_{e f f}+\frac{k_{(5)}^{4}}{12} \rho_{e f f}^{2}
$$

with

$$
\rho_{\text {eff }}=\frac{C}{a^{3}}+\frac{D /(\beta+1)}{a^{3(\beta+1)}} .
$$

The cosmological term $\Lambda_{(4)}$ is not constant, but evolves according to

$$
\Lambda_{(4)}=\frac{k_{(5)}^{4}}{6} \frac{\sigma_{0} D(-\beta)}{(\beta+1) a^{3(\beta+1)}}+\frac{k_{(5)}^{4}}{12} \frac{D^{2} \beta^{2}}{(\beta+1)^{2} a^{6(\beta+1)}} .
$$

\footnotetext{
${ }^{3}$ With this assumption $g$ is not constant.
} 


\subsubsection{Asymptotic behavior}

For $\beta=\gamma=0$, we recover the usual picture, i.e., $\rho=\bar{\rho}(1+z)^{3}, \sigma=\sigma_{0}$ and $\Lambda_{(4)}=0$. For $\beta<\gamma=0$, the vacuum term is initially negligible, which means that $\rho_{\text {eff }}$ approaches the typical matter density in FRW models and $(\sigma / \rho) \rightarrow 0$.

If $\sigma_{0}$ is positive, then the universe is in continuous expansion. When the vacuum term in (28) is so large that the ordinary matter contribution can be neglected, we find

$$
a^{3(\beta+1)} \approx \frac{k_{(5)}^{4} \sigma_{0} D(\beta+1)}{8} t^{2} .
$$

The corresponding "deceleration" parameter $q=-\ddot{a} a / \dot{a}^{2}$ is given by

$$
q \approx \frac{1+3 \beta}{2},
$$

which indicates that the expansion is accelerated for $\beta<-1 / 3$. At this stage of the evolution $G$ is constant and the cosmological term $\Lambda_{(4)}$ varies with time. Namely,

$$
8 \pi G \approx k_{(5)}^{4} \frac{\sigma_{0}}{6}, \quad \Lambda_{(4)} \approx \frac{3(\gamma-\beta)}{(\gamma+1)} H^{2}, \quad H \approx \frac{2}{3(\beta+1)} \frac{1}{t} .
$$

\subsubsection{The vacuum energy takes over}

We note that, for $\beta<0$, the first term in (28) decreases in time faster than the second one. Therefore, $D$ has to be chosen in such a way as to make the second term in (28) important at the right time to explain the observations. In order to do this, we find useful to introduce the auxiliary quantity $z_{e q}$. This is the redshift at which the vacuum and matter components in (28) become equal to each other ${ }^{4}$, i.e., $C / a^{3}=D /(\beta+1) a^{3(\beta+1)}$ at $a=a\left(z_{e q}\right)$. Since $a=\bar{a} /(1+z)$, where $\bar{a}$ is the present value of $a$, we obtain

$$
C=D \frac{\left(1+z_{e q}\right)^{3 \beta}}{(\beta+1) \bar{a}^{3 \beta}} .
$$

The appropriate $D$ follows from the evaluation of $\rho_{\text {eff }}$ today. We find

$$
\frac{D}{\bar{a}^{3(\beta+1)}}=\frac{(\beta+1) \bar{\rho}_{e f f}}{\left[1+\left(1+z_{e q}\right)^{3 \beta}\right]}
$$

where $\bar{\rho}_{\text {eff }}$ is the present value of the effective density.

We note that the case where $D=0$ and $C \neq 0$ is formally obtained from our equations by setting $z_{e q}=-1$, for any $\beta<0$. Similarly, the case where $C=0$ and $D \neq 0$ is formally attained in the limit $z_{e q} \rightarrow \infty$.

\section{Observational constraints on $\beta$}

Although the evolution equations (27)-(28) contain four constants: $C, D, \sigma_{0}$, and $k_{(5)}^{4}$, there are only two parameters in the model, viz., $\beta$ and $z_{e q}$. The aim of this section is to find out the physical restrictions on the parameter $\beta$.

With this goal, we express the relevant quantities in terms of these parameters and the present-value of the density of ordinary matter $\rho_{m}=C / a^{3}$. Let us start with the effective density (28). Using (33) and (34), it can be written as

$$
\rho_{e f f}=\bar{\rho}_{e f f} \frac{\left(1+z_{e q}\right)^{3 \beta}}{\left[1+\left(1+z_{e q}\right)^{3 \beta}\right]}(1+z)^{3}\left[1+\left(\frac{1+z}{1+z_{e q}}\right)^{3 \beta}\right] .
$$

\footnotetext{
${ }^{4}$ In order to avoid misunderstanding: the parameter $z_{e q}$ is not the redshift of transition from deceleration to acceleration, which we denote as $z_{T}$.
} 
The ratio of effective density to ordinary matter density is given by

$$
\frac{\rho_{e f f}}{\rho_{m}}=1+\left(\frac{1+z}{1+z_{e q}}\right)^{3 \beta} \text {. }
$$

Thus, at the present time

$$
\bar{\rho}_{e f f}=\bar{\rho}_{m} F\left(z_{e q}, \beta\right)
$$

where

$$
F\left(z_{e q}, \beta\right)=\frac{\left[1+\left(1+z_{e q}\right)^{3 \beta}\right]}{\left(1+z_{e q}\right)^{3 \beta}} .
$$

With this notation, we have $C=\bar{a}^{3} \bar{\rho}_{m}, D=\bar{a}^{3(\beta+1)}(F-1)(\beta+1) \bar{\rho}_{m}$. Consequently,

$$
\rho_{\text {eff }}=\rho_{m}\left[1+(F-1)(1+z)^{3 \beta}\right] .
$$

Thus, in our formulae the case of constant vacuum energy, for which $D=0$ and $\rho_{e f f}=\rho_{m}$, corresponds to $F=1$, for any $\beta<0$. For large redshifts $\rho_{e f f} \approx \rho_{m}$, while at the present time $\bar{\rho}_{e f f}=\bar{\rho}_{m} F$. We note that $F$ can be very large for large values of the parameter $Z_{e q}$.

\subsection{Positivity of $G$}

We now proceed to calculate $\sigma_{0}$. Evaluating (27) today, and using (37), we have

$$
3 \bar{H}^{2}=\frac{k_{(5)}^{4}}{6}\left[\sigma_{0} \bar{\rho}_{m} F\left(z_{e q}, \beta\right)+\frac{1}{2} \bar{\rho}_{m}^{2} F^{2}\left(z_{e q}, \beta\right)\right] .
$$

The constant $k_{(5)}^{4}$ is given by $k_{(5)}^{4}=48 \pi G / \sigma$. Thus, using (22), with $\gamma=0$, (34) and (38), we obtain

$$
k_{(5)}^{4}=\frac{48 \pi \bar{G}}{\left[\sigma_{0}-\beta \bar{\rho}_{m}(F-1)\right]},
$$

where $\bar{G}$ is the present value of the gravitational "constant" $G$. Feeding this expression back into (40) we obtain

$$
\sigma_{0}=\bar{\rho}_{m} \frac{\left[\bar{\Omega}_{m} F^{2} / 2+\beta(F-1)\right]}{\left[1-\bar{\Omega}_{m} F\right]}
$$

where $\bar{\Omega}_{m}$ is the present value of the mater density parameter $\Omega_{m}=8 \pi G \rho_{m} / 3 H^{2}$. The vacuum term (22) can be written as

$$
\sigma\left(z, z_{e q}, \beta\right)=\sigma_{0}-\beta \bar{\rho}_{m}(F-1)(1+z)^{3(\beta+1)} .
$$

Evaluating this equation today, we have

$$
\bar{\sigma}\left(z_{e q}, \beta, \bar{\Omega}_{m}\right)=\bar{\rho}_{m} \frac{\bar{\Omega}_{m} F[F / 2+\beta(F-1)]}{1-\bar{\Omega}_{m} F}
$$

We now calculate the constant $k_{(5)}^{4}$. For this we substitute (42) into (41). We get

$$
k_{(5)}^{4}\left(z_{e q}, \beta\right)=\frac{18 \bar{H}^{2}}{\bar{\rho}_{m}^{2}} \frac{\left[1-\bar{\Omega}_{m} F\right]}{[F / 2+\beta(F-1)] F} .
$$

We are now able to express general physical conditions on $\beta$. Since $8 \pi G=k_{(5)}^{4} \sigma / 6$, the positivity of $G$ demands $k_{(5)}^{4} \sigma>0$. Thus, for any given $\bar{\Omega}_{m}$ and $z_{e q}$, the allowed values of $\beta$, in (44) and (45), are those for which ${ }^{5}$

$$
k_{(5)}^{4}\left(z_{e q}, \beta, \bar{\Omega}_{m}\right)>0, \quad \bar{\sigma}\left(z_{e q}, \beta, \bar{\Omega}_{m}\right)>0 .
$$

\footnotetext{
${ }^{5}$ We note that $\sigma \geq \bar{\sigma}$. Also, the possibility $k_{(5)}^{4}\left(z_{e q}, \beta\right)<0$, and $\bar{\sigma}\left(z_{e q}, \beta, \bar{\Omega}_{m}\right)<0$ is excluded by the fact that $k_{(5)}^{2}$ in $(2)$ is a real number.
} 


\section{$4.2 \quad \dot{G} / G$}

More stringent restrictions on $\beta$ follow from observational constraints on the variation of $G$. In terms of the Hubble parameter, the time evolution of $G$ is usually written as $(\dot{G} / G)=g H$, where $g$ is a dimensionless parameter. In our model we have

$$
g=-3(\beta+1)\left(1-\frac{\sigma_{0}}{\sigma}\right) .
$$

We note that $g=0$ for $\beta=\gamma$ (i.e., $\beta=0$ for ordinary matter). From (42) and (44), we obtain the present value of $g$ as

$$
\bar{g}\left(z_{e q}, \beta, \bar{\Omega}_{m}\right)=-\frac{3 \beta(\beta+1)(F-1)\left(\bar{\Omega}_{m} F-1\right)}{\bar{\Omega}_{m} F[F / 2+\beta(F-1)]}
$$

The abundance of various elements as well as nucleosynthesis are used to put constraints on $g$ today. The present observational upper bound is ${ }^{6}$

$$
|\bar{g}| \leq 0.1 \text {. }
$$

Thus, the choice of $\beta$ has to guarantee the fulfillment of this condition. It clearly pushes the values of $\beta$ either toward $\beta \approx 0$ or $\beta \approx-1$.

\subsection{Deceleration parameter $q$}

In order to consider other observational constraints on $\beta$, let us introduce the deceleration parameter

$$
q=-\frac{\ddot{a} a}{\dot{a}^{2}}=-1+\frac{(1+z)}{H} \frac{d H}{d z},
$$

which in the case under study becomes

$$
q(z)=-1+\frac{3\left(\sigma_{0}+\rho_{e f f}\right)}{\left(2 \sigma_{0}+\rho_{e f f}\right)} \frac{\left[1+(\beta+1)(F-1)(1+z)^{3 \beta}\right]}{\left[1+(F-1)(1+z)^{3 \beta}\right]},
$$

where $\rho_{\text {eff }}(z)$ and $\sigma_{0}$ are given by (36) and (42), respectively. Evaluating this equation today, we have

$$
\bar{q}\left(z_{e q}, \beta, \bar{\Omega}_{m}\right)=-1+3 \frac{\left[F+\beta(F-1)-\bar{\Omega}_{m} F^{2} / 2\right][F+\beta(F-1)]}{F[F+2 \beta(F-1)]} .
$$

In the case where $\beta=\gamma$, the present-day acceleration (52) reduces to

$$
\bar{q}=2-3 \bar{\Omega}_{m} / 2 .
$$

In particular, for $\Omega_{m}=1$ we obtain $q=1 / 2$ as in the dust FRW cosmologies. We note that (53) is positive for any physical value of $\Omega_{m}$, which means that a brane-universe with constant vacuum energy must be slowing down its expansion ${ }^{7}$. However, for $\beta \neq \gamma$, this is no longer so; the vacuum energy $\sigma$ is now a dynamical quantity which changes this picture.

The choice of $\beta$ in (52) has to be consistent with recent measurements which indicate that the current universe is speeding up its expansion with an acceleration parameter which is roughly

$$
\bar{q}=-0.5 \pm 0.2 .
$$

\footnotetext{
${ }^{6} \mathrm{~A}$ comprehensive and updated discussion of the various experimental and observational constraints on the value of $g$ (as well as on the variation of other fundamental "constants" of nature) has recently been provided by Uzan [55]

${ }^{7}$ This contradicts the observational fact that the universe is speeding up and not slowing down (54).
} 


\subsection{The cosmological constant}

Finally, for the cosmological parameter $\Lambda_{(4)}$ at the present day we have

$$
\bar{\Lambda}_{(4)}=\xi\left(z_{e q}, \beta, \bar{\Omega}_{m}\right) \bar{H}^{2},
$$

where

$$
\xi\left(z_{e q}, \beta, \bar{\Omega}_{m}\right)=-\frac{3 \beta(F-1)\left[\bar{\Omega}_{m} F^{2}+\beta(F-1)\left(1+\bar{\Omega}_{m} F\right)\right]}{F[F+2 \beta(F-1)])} .
$$

Thus, $\bar{\Lambda}_{(4)}=0$ for $\beta=\gamma$, as expected. Otherwise, for $\beta \neq \gamma=0, \bar{\Lambda}_{(4)}$ has to be positive in order to explain the present acceleration.

\section{The auxiliary parameter $z_{e q}$}

We now turn our attention to parameter $z_{e q}$. Firstly, we confine the range of $z_{T}$, the redshift of transition from deceleration to acceleration, which is a solution of $q\left(z_{T}\right)=0$. Secondly, we set a lower bound on the value of the deceleration parameter today. Thirdly, we study in some detail the models with $\bar{\Omega}_{m}=0.3$. An interesting discovery here is the possibility of recollapsing models. In what follows we will only select $\beta$ from the range of values allowed by the requirements (46), (49) and (54).

\subsection{Transition from deceleration to acceleration}

There is plenty of observational evidence for a decelerated universe in the recent past, see e.g. [56-[57]. However, the dominance of the vacuum energy at some $z_{e q}>0$ does not guarantee the present acceleration of the universe. For this, the vacuum energy has to dominate long enough as to overcome the gravitational attraction produced by ordinary matter. However, for every fixed value of $\bar{\Omega}_{m}$, we can find a $z_{e q}^{\min }$ for which the transition from deceleration to acceleration occurs at some $z_{T}\left(0<z_{T}<z_{e q}^{\min }\right)$.

The transition is then guaranteed for $z_{e q}>z_{e q}^{m i n}$. A straightforward numerical study of (51), in the interval $\left(z_{e q}^{m i n}, \infty\right)$, reveals that $z_{T}$ is bounded above. Thus in our model, for every $\bar{\Omega}_{m}$ the transition from deceleration to acceleration occurs in a finite redshift interval

$$
z_{T}^{\min }<z_{T}<z_{T}^{\max }
$$

where the precise value of the lower and upper bounds depends on the density parameter for ordinary matter. The existence of an upper bound is an evidence that the dynamical influence of vacuum energy rapidly decreases for redshifts $z>z_{T}^{\max }$. We now proceed to show our numerical results for various values ${ }^{8}$ of $\bar{\Omega}_{m}$.

$\bar{\Omega}_{\mathbf{m}}=\mathbf{0 . 1}$ : We find that a transition from deceleration to acceleration is only possible if the vacuum energy starts to dominate in an epoch before $z_{e q}>z_{e q}^{m i n}=1.3$, otherwise the universe would be still today in a deceleration phase. The actual redshift of transition is $z_{T}=1.1$. A detailed investigation of $q\left(z_{T}\right)=0$, with $q$ given by (51), shows that $z_{T}<z_{T}^{\max }=1.81$ for $z_{e q}$ in the range $1.3<z_{e q}<\infty$, i.e., the transition occurs in the interval $z_{T}=(1.10,1.81)$. Thus, for the whole range of $z_{e q}$, we find that the redshift of transition from deceleration to acceleration, for $\bar{\Omega}_{m}=0.1$, is ${ }^{9}$

$$
z_{T} \approx 1.46 \pm 0.36
$$

\footnotetext{
${ }^{8} \mathrm{~A}$ reliable and definitive determination of $\bar{\Omega}_{m}$ has thus far eluded cosmologists. However, the observational data indicate that $\Omega_{\rho} \approx 0.1-0.3$ seem to be the most probably options.

${ }^{9}$ We note that these numbers are approximate; they depend on the specific choice of $\beta$ in the range that satisfies the conditions 46 and [49]. However, since $|\Delta \beta / \beta|$ is small and decreases with the increase of $z_{e q}$ (for an illustration see Table 1), the variation of these numbers is negligible and does not change the picture here.
} 
$\overline{\mathbf{\Omega}}_{\mathbf{m}}=\mathbf{0 . 2}$ : The transition is guaranteed for $z_{e q}>0.7$. It occurs in the redshift interval $z_{T}=(0.58,1.10)$, or equivalently

$$
z_{T} \approx 0.84 \pm 0.26
$$

and any value of $z_{e q}>0.7$.

$\bar{\Omega}_{\mathbf{m}}=\mathbf{0 . 3}$ : The vacuum contribution must start dominating at an epoch earlier than $z_{e q}=0.4$, otherwise there would be no enough time for a transition from a decelerating phase to an accelerating one today. For any $z_{e q}>0.4$, we find that the transition occurs at

$$
z_{T} \approx 0.48 \pm 0.20 .
$$

This is consistent with the value $z_{T} \approx 0.5$ given by Turner and Riess [57, and a little less than $z_{T} \approx 0.73$ provided by Perlmutter ${ }^{10}$ et al $[2]$.

The above discussion illustrates three things. Firstly, the fact that the redshift $z_{e q}$, at which the vacuum energy starts to dominate, depends on the mass density of ordinary matter; in a low matter density universe the vacuum energy starts to dominate before than in a universe with high matter density. Secondly, that the redshift of transition increases with the decrease of the matter density (58)-(60). Thirdly, that the vacuum effects only become important at the present epoch, making the transition a recent phenomenon.

\subsection{Lower bound on present deceleration (Upper bound on acceleration)}

The deceleration parameter today, which is given by (52), is an increasing function of $\beta$, for any fixed values of $z_{e q}$ and $\bar{\Omega}_{m}$. This means that its minimum value $\bar{q}_{\text {min }}$ is attained in the limit $\beta \rightarrow-1$.

Now, fixing $\bar{\Omega}_{m}$ and selecting an appropriate $\beta$ (as to satisfy the conditions discussed in the previous section) we find that $\bar{q}_{\text {min }}$ decreases for large values of $z_{e q}$. As an example consider the values presented in Table $1 ; \bar{q}_{\text {min }}$ increases for $z_{e q}<0.64$ and decreases for $z_{e q}>0.64$. Therefore, the lowest value of $\bar{q}_{\text {min }}$ is attained in the limit $z_{e q} \rightarrow \infty$. Thus, from (52) we get

$$
\bar{q}>-1+\frac{3}{2} \bar{\Omega}_{m},
$$

for any value of $z_{e q}$. For $\bar{\Omega}_{m}=0.3$, we find $\bar{q}>-0.55$, which is consistent with recent observations (54).

A similar analysis of (55) reveals that $\bar{\Lambda}_{(4)}$, the cosmological "constant" today, is bounded above. Namely,

$$
\bar{\Lambda}_{(4)}<3 \bar{H}^{2}\left(1-\bar{\Omega}_{m}\right),
$$

for any $z_{e q}$. Thus, if we take $\bar{\Omega}_{m}=0.3$, we get $\bar{\Lambda}_{(4)}<2.1 \bar{H}^{2}$. However, this is just a statement that in the present model $\bar{\Omega}_{m}+\bar{\Omega}_{\Lambda_{(4)}}<1$ in view of the quadratic correction in the generalized Friedmann equation.

\subsection{The model for $\bar{\Omega}_{m}=0.3$}

Current dynamical mass measurements suggest that the matter content of the universe adds up to $30 \%$ of the critical density ${ }^{11}$. According to (60) the transition from deceleration to acceleration occurs at a redshift $0.28<z_{T}<0.68$, which confirms the idea that the accelerated expansion of the universe is a recent phenomenon.

Thus, in our model, stars and galaxies with redshifts larger than $z \approx 0.68$ should reflect the kinematics of a decelerating expansion. This is compatible with galaxy formation, which can only take place if the gravitational attraction dominates a sufficiently long epoch over vacuum repulsion ${ }^{12}$. It also fits the observations of SN $1997 \mathrm{ff}$ at $z \approx 1.7$. This is the oldest and most distant type Ia supernova (SN Ia) discovered so far and provides direct evidence that the universe was decelerating before it began speeding up [60]. Other high redshift SNe known at $z=1.2$ (e.g.,

\footnotetext{
${ }^{10}$ It is encouraging that completely different models provide similar values for the redshift of transition from deceleration to acceleration. See for example 58

${ }^{11}$ Radiation $0.005 \%$, ordinary luminous baryonic matter $0.5 \%$, ordinary non-luminous baryonic matter $3.5 \%$ and exotic (non-baryonic) dark matter "observed" through their gravitational effects $26 \%$.

${ }^{12}$ For an updated analysis of the influence of dark energy on the first epoch of galaxy formation see, e.g., 59]
} 
SN 1999fv, SN 1998eq), $z=1$ (e.g., SN 1997ck, SN 1999fk) and $z=0.83$ (e.g., SN 1996cl) can provide a direct test for deceleration at the time of their explosion.

Also, from (60) it follows that galaxies with redshifts less that $z \approx 0.28$ should show evidence of an accelerating universe. Very-low redshift supernovae are crucial for reducing the uncertainty of the contemporary expansion rate.

We now proceed to study in more detail the parameters of the model for $\bar{\Omega}_{m}=0.3$, which is favored by observations. First of all, let us simplify the notation. To this end, we introduce the dimensionless parameters $\eta_{k}$ and $\eta_{\sigma_{0}}$ as

$$
\eta_{k} \equiv \frac{k_{(5)}^{4} \bar{\rho}_{m}^{2}}{18 \bar{H}^{2}}, \quad \eta_{\sigma_{0}} \equiv \frac{\sigma_{0}}{\bar{\rho}_{m}},
$$

in terms of which the generalized Friedmann equation (27) becomes

$$
H^{2}=\bar{H}^{2} \eta_{k}\left[\eta_{\sigma_{0}}+\frac{1}{2}\left(\frac{\rho_{e f f}}{\bar{\rho}_{m}}\right)\right]\left(\frac{\rho_{e f f}}{\bar{\rho}_{m}}\right),
$$

We note that

$$
\eta_{k} \eta_{\sigma_{0}} F+\frac{\eta_{k}}{2} F^{2}=1,
$$

which follows from (42) and (45), so that $H=\bar{H}$ today, as expected.

Examination of (44), (45) and (48) reveals that, for every given $z_{e q}$ and $\bar{\Omega}_{m}$, the adequate values of $\beta$, that satisfy the conditions (46) and (49), are spread over a small range. In that range $\bar{q}, \eta_{k}$ and $\eta_{\sigma_{0}}$ increase with $\beta$, while $\bar{\sigma}$ and $\bar{\Lambda}_{(4)}$ decrease with $\beta$. For example, if we take $z_{e q}=1$ and $\bar{\Omega}_{m}=0.3$, we find ${ }^{13} \beta=-0.989 \pm 0.011$. The corresponding $\bar{q}$ increases from $\bar{q}=-0.46$ for $\beta \approx-1$ to $\bar{q}=-0.35$ for $\beta=-0.978$. The other quantities undergo a relatively smaller change, viz.,

$$
\eta_{k}=0.056_{-0.002}^{+0.002}, \quad \eta_{\sigma_{0}}=-2.390_{-0.046}^{+0.049}, \quad \frac{\bar{\sigma}}{\bar{\rho}_{m}}=5.343_{-0.210}^{+0.196}, ; \quad \frac{\bar{\Lambda}_{(4)}}{\bar{H}^{2}}=1.923_{-0.094}^{+0.087} .
$$

For the redshift of transition from deceleration to acceleration we find

$$
z_{T}=(0.335,0.298,0.279) \text { for } \beta=(-0.999,-0.985,-0.978) \text { respectively. }
$$

In Table 1, we illustrate the cosmological parameters for various values of $z_{e q}$ and $\bar{\Omega}_{m}=0.3$. For the sake of simplicity, we omit their small change over the range of $\beta$ and present their mean values only ${ }^{14}$, corresponding to the average of $\beta$. Like we have said before the vacuum contribution must start dominating at an epoch earlier than $z_{e q}=0.4$, otherwise the universe would be still in a decelerating phase.

\begin{tabular}{|c|c|c|c|c|c|c|c|c|}
\hline \multicolumn{7}{|c|}{ Table 1: Cosmological parameters for $\Omega_{m}=0.3$ fitting $|g|<0.1$} \\
\hline$z_{e q}$ & $-1<\beta<$ & $\eta_{k}$ & $\left(\bar{\sigma} / \bar{\rho}_{m}\right)$ & $\bar{q}$ & $\eta_{\sigma_{0}}$ & $H \bar{t}$ & $\left(\Lambda_{(4)} / H^{2}\right)$ & $\left.z_{T}=z_{q=0}\right)$ \\
\hline \hline 0.4 & -0.840 & 0.030 & 10.052 & $(-0.49,-0.38)$ & 7.724 & 0.743 & 1.853 & 0.378 \\
\hline 0.5 & -0.949 & 0.063 & 4.791 & $(-0.46,-0.35)$ & 1.602 & 0.691 & 1.915 & 0.293 \\
\hline 0.6 & -0.963 & 0.070 & 4.281 & $(-0.45,-0.34)$ & 0.364 & 0.681 & 1.912 & 0.277 \\
\hline 0.7 & -0.970 & 0.070 & 4.298 & $(-0.45,-0.34)$ & -0.427 & 0.682 & 1.915 & 0.278 \\
\hline 0.8 & -0.974 & 0.066 & 4.529 & $(-0.45,-0.35)$ & -1.097 & 0.686 & 1.919 & 0.286 \\
\hline 0.9 & -0.976 & 0.061 & 4.885 & $(-0.46,-0.35)$ & -1.737 & 0.693 & 1.920 & 0.297 \\
\hline 1.0 & -0.978 & 0.056 & 5.343 & $(-0.46,-0.35)$ & -2.390 & 0.701 & 1.923 & 0.311 \\
\hline 1.1 & -0.979 & 0.051 & 5.879 & $(-0.47,-0.35)$ & -3.073 & 0.708 & 1.923 & 0.324 \\
\hline 1.2 & -0.979 & 0.046 & 6.482 & $(-0.48,-0.35)$ & -3.796 & 0.716 & 1.916 & 0.336 \\
\hline 1.5 & -0.981 & 0.034 & 8.768 & $(-0.49,-0.34)$ & -6.309 & 0.738 & 1.903 & 0.375 \\
\hline 1.7 & -0.982 & 0.028 & 10.673 & $(-0.50,-0.32)$ & -8.317 & 0.751 & 1.887 & 0.398 \\
\hline
\end{tabular}

\footnotetext{
${ }^{13}$ For $z_{e q}=1$ and $\bar{\Omega}_{m}=0.3$, any $\beta$ in the interval $(-1,-0.65)$ satisfies $k_{(5)}^{4}>0$ (or $\eta_{k}>0$ ) and $\bar{\sigma}>0$. However, the condition $|g|<0.1$ narrows down this interval to $(-1,-0.978)$.

${ }^{14}$ See footnote 9
} 
The second column in Table 1 gives the range of $\beta$, for different values of $z_{e q}$, that satisfy the requirements $k_{(5)}^{4}>0, G>0$ and $|g|<0.1$. This is illustrated in columns 3 and 4 by the positive values of $\eta_{k}$ and $\left(\bar{\sigma} / \bar{\rho}_{m}\right)$, respectively.

For these values, it turns out that the acceleration of the universe today is not an additional constraint or assumption. On the contrary, it is a consequence of the model and the observational requirement $|g|<0.1$. The significant point here is that the results, which are presented in column 5 , are consistent with current observations (54). Besides, our model narrows down the possible values of $q$ today (61).

For larger values of $z_{e q}$, say $z_{e q}>1.8$, the requirements (46) and (49) are automatically satisfied if $\beta$ is selected in the range $(-1,-0.983)$. However, the cosmic acceleration (54) is not a consequence of the model (as it is for $\left.z_{e q} \leq 1.8\right)$ but an observational constraint which restricts the values of $\beta$ even further.

Column 6 presents the dimensionless parameter $\eta_{\sigma_{0}}$. It changes sign for $z_{e q} \approx 0.64$. Therefore, there are two kinds of models:

(i) Ever-expanding models. These are the ones with $z_{e q}<0.64$, for which $\eta_{\sigma_{0}}>0$. After a long matter-dominated phase of deceleration, the universe is accelerating today and will continue that way in the future ${ }^{15}$.

(ii) Recollapsing models. For $z_{e q}>0.64$, we have $\eta_{\sigma_{0}}<0$. After a long matter-dominated phase of deceleration, the universe is accelerating today, but deceleration comes back at some point in the future ${ }^{16}$. From (64) it follows that the expansion must come to an end before the universe starts contracting. Setting $H=0$ we can get $a_{r e c}$, the size of the universe at the moment of recollapse. Namely,

$$
\left(\frac{\bar{a}}{a_{r e c}}\right)^{3}+(F-1)\left(\frac{\bar{a}}{a_{r e c}}\right)^{3(\beta+1)}+2 \eta_{\sigma_{0}}=0 .
$$

Clearly, in the case under consideration the second term amply dominates over the first one. Therefore, from (68) we get

$$
a_{\text {rec }} \approx \bar{a}\left(\frac{F-1}{2\left|\sigma_{0}\right|}\right)^{1 / 3(\beta+1)}, \quad \beta \neq-1 .
$$

The reason for the recollapse is that the four dimensional cosmological constant changes its sign sometime in the far future. Using (29) and (69), we find

$$
a_{\Lambda_{(4)}=0} \approx|\beta|^{1 / 3(\beta+1)} a_{\text {rec }} .
$$

As an example, we use the data for $z_{e q}=0.7$. We find

$$
a_{r e c} \approx 4.44 \times 10^{16} \bar{a}, \quad a_{\Lambda_{(4)}} \approx 0.71 a_{r e c} .
$$

The good news is that we are nowhere near the recollapse!

The age of the universe $\bar{t}$, in terms of the current value of the Hubble "constant" $\bar{H}$, is presented in the seventh column. The main feature here is that the universe is older than the FRW counterpart for which $\bar{H} \bar{t}=2 / 3$. To put the discussion in perspective, we notice that in braneworld models (with $\Lambda_{(4)}=0$ ) the universe is younger than in the standard FRW cosmologies ${ }^{17}$. The age of the universe increases with $z_{e q}$. As an example, if we take $z_{e q}=100$ (for this value the crossover point is $z_{T}=0.67$ ), we find $\bar{H} \bar{t}=0.96$.

Finally, the redshift of transition is specified in column 9. For the values considered here the crossover takes place at $z_{T} \sim 0.28-0.4$. However, it can occur even earlier if the vacuum contribution starts to dominate for larger values of $z_{e q}$, in which case the range for $z_{T}$ extends to $0.28<z_{T}<0.68$. This redshift interval coincides with the time of explosions of a number of SNe Ia known today. For example, SN 1988 at $z=0.31$, SN 1992bi at $z=0.46$, SN 1995K

\footnotetext{
${ }^{15}$ In order to avoid misunderstanding, let us mention that the equation $\sigma_{0}\left(z_{e q}, \beta\right)=0$ does have a solution for $z_{e q}=(0.4,0.5,0.6,0.64)$ and $\beta=(-0.67,-0.72,-0.83,-0.96)$, respectively. But these values of $\beta$ do not satisfy the physical conditions (46), 49) and (54). Therefore they are excluded here. More precisely, the only models with $\sigma_{0}=0$ that satisfy physical conditions are those with $z_{e q}$ in the interval $(0.642,0.643)$ and $-1<\beta<-0.967$.

${ }^{16}$ For $0.64<z_{e q}<\infty$, using (51) we find that equation $q\left(z_{T}\right)=0$ has two set of solutions. One for positive $z$, which is given by 60 , and another one for negative $z$, namely $-1<z_{T}<-0.22$. The second solution corresponds to the future, when the size of the universe is $a>1.28 \bar{a}$.

${ }^{17}$ For example, for a dust-filled universe without cosmological term, we find $\bar{t}_{\text {brane }}=0.363 \bar{H}^{-1}$ in brane models, while $\bar{t}_{F} R W=2 \bar{H}^{-1} / 3$ in FRW models.
} 
at $z=0.48$ and SN 1995 at $z=0.66$. Therefore, they can provide crucial information to reduce the uncertainty in the transition between deceleration and acceleration.

For completeness, we mention that the cosmological constant in the bulk, using (26) and (63), can be written as

$$
\Lambda_{(5)}=-\frac{\eta_{\sigma_{0}}^{2} \sqrt{\eta_{k}}}{\sqrt{2}} \bar{H} \bar{\rho}_{m}
$$

Thus, we can "predict" the value of $\Lambda_{(5)}$ by means of measurements in $4 D$.

\section{Discussion and conclusions}

An important distinction between general relativity and braneworld theory is that the cosmological term in the first is put by "hand" while in the second it is determined by the solution in the bulk through Israel's boundary conditions.

A variable vacuum energy can generally occur as a consequence of embedding our universe as a brane in a fivedimensional bulk with non-static extra dimensions. There are a number of known solutions in $5 D$ for which the vacuum density on the brane decreases as an inverse power of the scale factor, similar (but at different rate) to the power law in FRW-universes of general relativity.

In this paper, we devoted our attention to spatially flat, homogeneous and isotropic, brane-universes where the vacuum density decays as in (22). The model contains two parameters, viz., $\beta$ and $z_{\text {eq }}$.

If $\beta=\gamma$, then $\Lambda_{(4)}=0, G$ is a universal constant and $\bar{q}=2-3 \bar{\Omega}_{m} / 2$, in the dust dominated era. Thus fixing $\Omega_{m}$ today also fixes $q$. Notice that $q$ is positive for any physical value of $\Omega_{m}$, meaning that a brane-universe with constant vacuum energy must be slowing down its expansion.

However, for $\beta \neq \gamma$, this is no longer so; the vacuum energy is now a dynamical quantity which changes this picture. In fact, $\mathrm{G}$ as well as $\Lambda_{(4)}$ become functions of time and the deceleration parameter decreases from $q_{\gamma} \approx 3 \gamma+2$ at the beginning of the universe to $q \rightarrow-1+3 \Omega_{m} / 2$ at the present time. Thus, for any matter with $\gamma>-2 / 3, q$ is positive at the beginning and negative today, because currently $\bar{\Omega}_{m}<2 / 3$.

Our model predicts that the transition from deceleration to acceleration occurred only recently, for $z_{T}<0.68$, but not later than $z_{T} \approx 0.28$, regardless of the specific value of $z_{e q}$. Therefore, early structure formation, from small density inhomogeneities, is not affected.

If the domination of the vacuum is recent, i.e., $z_{e q}<1.8$, then the observed accelerated cosmic expansion is, not a condition but, a consequence of our model. The truth is that we have no observational or theoretical reasons to believe that vacuum started to dominate before $z \sim 1$. Consequently, we can safely declare that our model predicts the present acceleration of the universe.

What is important here is that the predicted values for $\bar{q}$ are consistent with observations and allow us to narrow down the experimental uncertainty in the current data; from (54) to

$$
\bar{q}=-0.41 \pm 0.09 .
$$

This may help in observations for an experimental/observational test of the model.

Let us notice that an alternate way to write the effective density (39) is

$$
\rho_{e f f}=\rho_{m}+\frac{(F-1)}{\bar{\rho}_{m}^{(n-1)}} \rho_{m}^{n}
$$

where $n=(\beta+1)$. This peculiar notation allows us to make contact between our ever-expanding models (those with $\left.\sigma_{0}>0\right)$ and Cardassian models. Indeed, for $\sigma_{0} \gg \rho_{e f f} / 2$ the second term in (27) can be neglected ${ }^{18}$. In this approximation, the generalized Friedmann equation (27) can be written as

$$
H^{2} \approx A \rho_{m}+B \rho_{m}^{n},
$$

${ }^{18}$ This is clearly satisfied for $z_{e q} \approx 0.4$ during the recent epoch of accelerated expansion which started at $z \approx 0.38$. See Table 1 
with $n=(\beta+1)$ and

$$
A=\frac{\bar{H}^{2} \eta_{k} \eta_{\sigma_{0}}}{\bar{\rho}_{m}}, \quad B=\frac{\bar{H}^{2} \eta_{k} \eta_{\sigma_{0}}(F-1)}{\bar{\rho}_{m}^{n}} .
$$

Equation (75) is similar to the one used in the so-called Cardassian models [40]. It differs from the usual Friedmann equation of general relativity by the addition of the extra term $\rho_{m}^{n}$. Therefore, based on (75), one can interpret Cardassian expansion as the low energy limit of the brane model discussed here. Freese and Lewis [40] and Gondolo and Freese [41] suggest that the extra term may arise as a consequence of embedding our universe as a brane in extra dimensions. This is exactly what we have here.

Nonetheless, there is a big difference in the understanding of the extra term. In the Cardassian model, by assumption, there is no vacuum contribution and the new term may come from some (yet unknown) modified Einstein equations. In our model the extra term is a manifestation of the variation of vacuum, in response to the time evolution of the extra dimension

This difference leads to distinct requirements on $n$ (or $\beta$ ). In Cardassian models the most stringent requirements on $n$ demand $n<0.4$ (equivalently, $\beta<-0.6$ ). While, in our model the parameter $\beta$ depends on $\bar{\Omega}_{m}$ and is severely restricted by physical conditions $k_{(5)}^{4}>0, G>0$ and $|g|<0.1$. Indeed, for $\bar{\Omega}_{m}=0.3$ we found $-1<\beta<-0.84$ (equivalently, $0<n<0.16$ ).

In order to get another interpretation we write (75) as

$$
H^{2} \approx \bar{H}^{2}\left[\Omega_{M}(1+z)^{3}+\Omega_{X}(1+z)^{3\left(w_{X}+1\right)}\right],
$$

with $w_{X}=\beta, \Omega_{M}=\eta_{k} \eta_{\sigma_{0}}$ and $\Omega_{X}=\eta_{k} \eta_{\sigma_{0}}(F-1)$. In this approximation $F \approx\left(\eta_{k} \eta_{\sigma_{0}}\right)^{-1}$, so that $\Omega_{M}+\Omega_{X} \approx 1$. The above expression is simikar to the Friedmann equation in cosmological models with quintessence, where the dark energy component is characterized by the equation of state $w_{X}=p_{X} / \rho_{X}$.

Thus, if the vacuum energy only started to dominate recently, at $z_{e q} \sim 0.4-0.6$, then at low energy limit there is a correspondence between our ever-expanding as well as the Cardassian and quintessence models, although they are entirely different in philosophy.

However, if the vacuum energy started to dominate earlier, say at $z_{e q}>0.64$, then $\sigma_{0}$ is negative and the quadratic term in (27) cannot be disregarded. As a consequence our model (for $z_{e q}>0.64$ ) is entirely different from Cardassian and quintessence models. In particular, it predicts that our universe will collapse in the future.

We would like to finish this work with the following comments. The whole analysis in this paper is independent of any particular solution used in the five-dimensional bulk. This is a great virtue of brane-world models as noted at the end of Section 2. However, one could still ask whether the universe described here can be embedded in a fivedimensional bulk. The answer to this question is positive. For example, they can be embedded in five-dimensional "wave-like" cosmologies of the type discussed in 61]. If in equation (38) of 61] we take variable $\sigma$ as here in (22), then the scale factor $a$ for such wave-like models is governed by an equation which is identical to (25) in this paper.

It is important to mention that the ratio $(\dot{\Phi} / \Phi)$ appears in different contexts, notably in expressions concerning the variation of rest mass [62]- 63], electric charge [64 and variation of the gravitational "constant" $G$ [65, [66]. Therefore, we have a scenario where the observed cosmic acceleration is just one piece in the dynamical evolution of an universe where the so-called fundamental "constants" are evolving in time. Braneworld scenario may provide us a theoretical framework to unify all these, apparently, separated phenomena as different consequences in $4 D$ of the time evolution of the extra dimension. This is a new step toward understanding how the universe works.

Note added in proof: While different alternative explanations are given to explain the current acceleration of the universe [21]-44], the dimming of the supernovae could be explained on the basis of axion physics 67.

\section{References}

[1] A.G. Riess et al., Supernova Search Team Collaboration, Astron. J., 116, 1009 (1998); astro-ph/9805201

[2] S. Perlmutter et al., Supernova Cosmology Project Collaboration, Astrophys. J., 517, 565 (1999); astro-ph/9812133 
[3] Andrew R Liddle, New Astron.Rev., 45, 235(2001), astro-ph/0009491.

[4] N. Seto, S. Kawamura and T. Nakamura, Phys.Rev.Lett. 87, 221103(2001), astro-ph/0108011

[5] R. A. Knop et al, Astrophys. J., 598, 102(2003); astro-ph/0309368

[6] J.L. Tonry et al., Astrophys. J. 594, 1 (2003); astro-ph/0305008

[7] A.T. Lee et al, Astrophys. J., 561, L1(2001); astro-ph/0104459

[8] R. Stompor et al, Astrophys. J., 561, L7(2001); astro-ph/0105062

[9] N.W. Halverson et al, Astrophys. J., 568, 38(2002); astro-ph/0104489

[10] C.B. Netterfield et al, Astrophys. J., 571, 604(2002); astro-ph/0104460

[11] C. Pryke, et al., Astrophys. J., 568, 46(2002); astro-ph/0104490

[12] D.N. Spergel et al., Astrophys. J.Suppl., 148, 175 (2003); astro-ph/0302209

[13] J. L. Sievers, et al., Astrophys. J., 591, 599(2003); astro-ph/0205387

[14] P. J. E. Peebles and B. Ratra, Rev.Mod.Phys. 75, 559(2003), astro-ph/0207347

[15] T. Padmanabhan, Phys.Rept. 380, 235(2003), hep-th/0212290

[16] I. Zlatev, L Wang and P. J. Steinhardt, Phys.Rev.Lett. 82, 896(1999), astro-ph/9807002

[17] C. Armendariz, V. Mukhanov, P. J. Steinhardt, Phys.Rev.Lett. 85, 4438(2000), astro-ph/0004134

[18] R.R. Caldwell, R. Dave, P. J. Steinhardt, Phys.Rev.Lett. 80, 1582(1998), astro-ph/9708069

[19] S.E. Deustua, R. Caldwell, P. Garnavich, L. Hui, A. Refregier, "Cosmological Parameters, Dark Energy and Large Scale Structure", astro-ph/0207293.

[20] T.Padmanabhan, "Cosmic inventory of energy densities: issues and concerns", IUCAA preprint 59/2001; gr-qc/0112068

[21] R. R. Caldwell, M. Kamionkowski, N. N. Weinberg, Phys.Rev.Lett. 91, 071301(2003), astro-ph/0302506.

[22] R.R. Caldwell, Phys.Lett. B545, 23(2002), astro-ph/9908168

[23] S. Nojiri and S. D. Odintsov, Phys.Lett. B562, 147(2003), hep-th/0303117, Phys.Rev. D68, (2003) 123512, hep-th/0307288.

[24] H. Stefancic, Phys.Lett., B586, 5(2004), astro-ph/0310904 Eur.Phys. J., C36, 523(2004). astro-ph/0312484

[25] S. M. Carroll, V. Duvvuri, M. Trodden and M. S. Turner, Phys.Rev., D70, 043528(2004); astro-ph/0306438

[26] G. Dvali and M. S. Turner, "Dark Energy as a Modification of the Friedmann Equation", astro-ph/0301510.

[27] S. Bayin, Int. J. of Mod. Phys. D11, 1523(2002), astro-ph/0211097

[28] D.F. Mota and J.D. Barrow, Mon. Not. Roy. Astron. Soc., 349 281(2004), astro-ph/0309273 Phys.Lett. B581, 141(2004), astro-ph/0306047

[29] Yungui Gong and Chang-Kui Duan, Class.Quant.Grav., 21, 3655(2004) ; gr-qc/0311060

[30] S. Capozziello, S. Carloni, A. Troisi, "Recent Research Developments in Astronomy and Astrophysics"RSP/AA/21-2003; astro-ph/0303041 
[31] S.M. Carroll, V. Duvvuri, M. Trodden and M. S. Turner, Phys.Rev., D70 043528(2004); astro-ph/0306438

[32] A.D. Dolgov, M. Kawasaki, Phys.Lett., B573, 1(2003); astro-ph/0307285.

[33] D. A. Easson, "Cosmic Acceleration and Modified Gravitational Models", astro-ph/0411209

[34] A. Lue, G. Starkman, Phys.Rev. D67, 064002(2003), astro-ph/0212083.

[35] C. Deffayet, G. Dvali and G. Gabadadze, Phys.Rev. D65 044023(2002), astro-ph/0105068.

[36] C. Deffayet, S. J. Landau, J. Raux, M. Zaldarriaga and P. Astier, Phys.Rev. D66, 024019(2002), astro-ph/0201164

[37] V. Gorini, A. Kamenshchik and U. Moschella, Phys.Rev. D67 063509(2003), astro-ph/020939

[38] R. Neves, C. Vaz, Phys.Rev. D68, (2003) 024007, hep-th/0302030 Phys.Lett. B568, 153(2003), hep-th/0304266

[39] M.C. Bento, O. Bertolami, A.A. Sen, Phys.Lett. B575, 172(2003), astro-ph/0303538 Gen.Rel.Grav. 35, 2063(2003), gr-qc/0305086 Phys.Rev. D66, 043507(2002), gr-qc/0202064

[40] K. Freese and M. Lewis, Phys.Lett. B540 1(2002), astro-ph/0201229

[41] P. Gondolo and K. Freese, Phys.Rev. D68, 063509(2003), hep-ph/0209322

[42] R. G. Vishwakarma, "In Quest of a True Model of the Universe", astro-ph/0404371 Has received Honorable Mention in the essay competition 2004 sponsored by the Gravity Research Foundation.

[43] R. G. Vishwakarma, Mon.Not.Roy.Astron.Soc. 345, 545(2003); astro-ph/0302357

[44] R. G. Vishwakarma and Parampreet Singh, Class.Quant.Grav. 20, 2033(2003); astro-ph/0211285.

[45] T. Shiromizu, K. Maeda and M. Sasaki, Phys. Rev. D62, 02412(2000), gr-qc/9910076

[46] Roy Maartens, "Geometry and dynamics of the brane-world", Reference Frames and Gravitomagnetism, ed. J Pascual-Sanchez et al. (World Sci., 2001), p93-119, gr-qc/0101059.

[47] P. Binetruy, C. Deffayet, U. Ellwanger and D. Langlois, Phys.Lett. B477 285(2000), hep-th/9910219

[48] J. Ponce de Leon, Mod.Phys.Lett. A17, 2425(2002), gr-qc/0207001

[49] J. Ponce de Leon, Class.Quant.Grav. 20, 5321(2003), gr-qc/0305041

[50] J. Ponce de Leon, "Accelerated expansion from braneworld models with variable vacuum energy" To appear in GRG, gr-qc/0401026.

[51] D. Huterer and M. S. Turner, Phys.Rev. D60, 081301(1999), astro-ph/9808133.

[52] T. Nakamura and T. Chiba, Mon.Not.Roy.Astron.Soc. 306, 696(1999), astro-ph/9810447

[53] B.F. Gerke and G. Efstathiou, Mon.Not.Roy.Astron.Soc. 335, 33(2002), astro-ph/0201336.

[54] P. de Bernardis et al, Nature 404, 955(2000).

[55] J.P. Uzan, Rev. Mod. Phys. 75, 403(2003), hep-ph/0205340

[56] A.G. Riess et al, Astrophys.J. 560, 49(2001), astro-ph/0104455

[57] M. S. Turner and A. Riess "Do SNe Ia Provide Direct Evidence for Past Deceleration of the Universe?" astro-ph/0106051 
[58] S. Bayin, "Missing Mass, Dark Energy and the Acceleration of the Universe. Is Acceleration Here to Stay?", astro-ph/0410710

[59] J. S. Alcaniz and J. A. S. Lima, Astrophys. J., 550, L133(2001); astro-ph/0101544.

[60] A. G. Riess et al., Astrophys. J., 560, 49(2001); astro-ph/0104455

[61] J. Ponce de Leon, Gen.Rel.Grav., 36, 923(2004); gr-qc/0212058

[62] J. Ponce de Leon, Int.J.Mod.Phys. D12, 757(2003) gr-qc/0209013

[63] J. Ponce de Leon, Gen.Rel.Grav., 36, 1335(2004); gr-qc/0310078.

[64] J. Ponce de Leon, Gen.Rel.Grav., 35, 1365(2003); gr-qc/0207108

[65] V.N. Melnikov and V.D. Ivashchuk, "Problems of $G$ and multidimensional models" Report-no: IGC-PFUR01/2002, gr-qc/0208021.

[66] V.N. Melnikov, "Gravity as a Key Problem of the Millennium". Proc. 2000 NASA/JPL Conference on Fundamental Physics in Microgravity, CD-version, NASA Document D-21522, 4.1-4.7(2001), (Solvang, CA, USA), gr-qc/0007067

[67] C. Csaki, N. Kaloper and J. Terning, Phys.Rev.Lett., 88, 161302(2002); hep-ph/0111311 